\title{
PROPUESTA INICIAL PARA EL DESARROLLO DE UNA METODOLOGÍA QUE PERMITA LA IMPLEMENTACIÓN IPTVv6 SOBRE SISTEMAS IMS
}

Jean Paul Granados Cárdenas

Especialista en Teleinformática

Instituto Caro y Cuervo

jean.paul.granados@gmail.com

Bucaramanga, Colombia

\section{Danilo Alfonso López Sarmiento}

Magister en Ciencias de la Información y las Comunicaciones

Docente planta de la Universidad Distrital Francisco José de Caldas dalopezs@udistrital.edu.co

Bogotá, Colombia

\section{César Augusto Hernández Suárez}

Magister en Ciencias de la Información y las Comunicaciones

Docente planta de la Universidad Distrital Francisco José de Caldas cahernandezs@udistrital.edu.co Bogotá, Colombia

Tipo: Artículo de investigación

Fecha de Recepción: Junio 7 de 2013

Fecha de Aceptación: Agosto 6 de 2013

\section{PRELIMINARY PROPOSAL FOR THE DEVELOPMENT OF A METHODOLOGY THAT ALLOWS THE IPTVV6 IM- PLEMENTATION ON IMS SYSTEMS}

\begin{abstract}
Digital television has continuously evolved since its digital terrestrial appearance (DVB-T), passing through the satellite standard (DVB-S) until reaching its current presentation known as IPTV (Internet Protocol Television), which offers many multimedia services such as voice, video and data, that are conveyed with certain levels of quality of service, security, interactivity and reliability through the use of the TCP/IP protocol stack. In this sense the article provides to the academic community a preliminary methodological proposal which focuses on working as a guidance in the implementation of such systems on a platform of free distribution, known as IMS (IP Multimedia Subsystem) for IPv6 type local area networks.
\end{abstract}

Key words: IPTV, IMS, SIP, RTP, IPv6.

\section{RESUMEN}

La televisión digital ha evolucionado continuamente desde su presentación digital terrestre (DVB-T), pasando por el estándar satelital (DVB-S) hasta llegar a su presentación actual conocida como IPTV (Internet Protocolo Television) que ofrece múltiples servicios multimedia tales como voz, video y datos, transportados con ciertos niveles de calidad de servicio, seguridad, interactividad y confianza gracias a la utilización de la pila de protocolos TCP/IP. En tal sentido el artículo pone a disposición de la comunidad académica una propuesta metodológica inicial que pretende servir de guía en la implementación de este tipo de sistemas sobre una plataforma de libre distribución conocida como IMS (IP Multimedia Subsystem) para redes de área local tipo IPv6.

Palabras claves: IPTV, IMS, SIP, RTP, IPv6. 


\section{INTRODUCCIÓN}

En Colombia los ISP ofrecen planes de datos, voz y televisión digital en un solo paquete (triple play), pero ninguno de estos posee una plataforma bajo la arquitectura IMS en redes IPv6, la cual requiere de una capa de control y de aplicación adicional, separados y desarrollados especialmente para manejar servicios de televisión digital (Middleware); pero que tiene la ventaja de ofrecer una mayor cantidad de prestaciones a muy bajo precio por ser de libre distribución. Por tal motivo el documento pretende plasmar los principales elementos que permitirían eventualmente el normal desarrollo y configuración de IPTV sobre IMS con soporte para IPv6, a partir de la exploración temática de las diferentes arquitecturas y protocolos que se verían involucrados, y a partir de allí poder incluirlos en una metodología que establezca los requerimientos de hardware y software necesarios para implementar IPTV6 en IMS. El artículo en la sección 2 detalla los conceptos, tipos de arquitecturas y características de IPTV; en la sección 3 se realiza una exploración del funcionamiento y los protocolos que incluye la arquitectura IPTV sobre IMS, para luego desarrollar una metodología que establezca la forma más adecuada de implementarse que aparece en la sección 4.

\section{ARQUITECTURA DE IPTV}

\subsection{Definición de IPTV}

IPTV es un sistema donde el servicio de televisión digital es entregado sobre la red IP de forma segura y donde el operador puede garantizar calidad de servicio. Tiene la capacidad de incluir prestaciones como VoIP y datos o acceso Web. Desde la perspectiva de los ISP's, este concepto abarca la adquisición, procesamiento y entrega segura del contenido de video basada en IP. Esta tecnología ha evolucionado desde la IPTV sin soporte en NGN hasta una arquitectura de IPTV basada en IMS [5] [12].

\subsection{Características de IPTV}

IPTV permite a los proveedores de servicios entregar una amplia gama de aplicaciones entre las que se encuentran [5]:

- Televisión con calidad de alta definición (HDTV) y de definición estándar (SDTV).

- Juegos interactivos.

- Alta velocidad de navegación en Internet.

- Desplazamiento en el tiempo. IPTV en combinación con una grabadora de video digital permite el movimiento fuera de secuencia del contenido multimedia.

- Personalización, a través de una comunicación bidireccional extremo a extremo permitiendo decidir lo que se quiere ver cuando quiera verlo.

- Exigencias reducidas de anchos de banda cuando se utiliza un formato de compresión como el H.264 y una estructura de envío tipo multicast.

- Accesibilidad a contenidos en múltiples dispositivos mediante la visualización de estos desde computadores, televisores y dispositivos móviles [5].

\section{ARQUITECTURA DE IPTV SOBRE IMS}

IMS fue introducido por 3GPP, como un subsistema dentro de las redes móviles de tercera generación con el fin de proveer y administrar productos multimedia sobre redes de conmutación de paquetes. La propuesta de IMS fue tan novedosa para manejar los servicios multimedia que fue adoptado por la arquitectura IPTV. IMS establece una comunicación de forma estandarizada, controlando los distintos componentes o entidades mediante el protocolo SIP y permitiendo la convergencia de distintos tipos de redes de acceso [5].

\subsection{Ventajas de implementar IPTV sobre IMS}

Las funcionalidades de IMS permite que la arquitectura IPTV ofrezca importantes características y servicios novedosos (a muy bajos precios contrario a lo que sucede con otro tipo de tecnologías) como [9] [1] [13] [4]:

- Convergencia tanto de redes fijas como móviles. 
- Implementación de QoS y QoE de extremo a extremo.

- Establecimiento y administración de la sesión de comunicación usando el protocolo SIP.

- Registro, control y autenticación de manera integrada y centralizada mediante la base de datos HSS (Home Subscriber Server) dentro del núcleo IMS.

- Personalización de los servicios mediante el manejo de una base de datos centralizada (HSS).

- Administración de manera fácil y central de las suscripciones de los usuarios.

- Servicios de presencia, chat y mensajería.

- Movilidad a través del servicio de roaming.

- Solución unificada de cobro y facturación.

- Convergencia de voz, video, datos y servicios de telefonía móvil.

- Adaptación y personalización de contenidos.

- Servicios de juego.

\subsection{Estructura funcional de IPTV sobre IMS}

En la figura 1 se muestra la arquitectura de IPTV basada en IMS como lo definió la ETSI TISPAN. Esta arquitectura se compone de cinco bloques, que incluyen las funciones de transporte, el núcleo IMS, el servidor de aplicación de IPTV (IPTVAS), el equipo terminal (el UE) y el bloque del Servidor de distribución multimedia (streaming server), este último formado por dos elementos que son el control y entrega del contendido. El servicio de descubrimiento es realizado mediante los servidores de aplicación SIP conocidos como (SDF o funciones de detección de servicio), y quien es el que proporciona la información acerca de la función de selección del servicio (SSF) requerido por el usuario. La función de control de servicio (SCF) es responsable de atender las solicitudes basadas en el protocolo SIP entregada sobre la interfaz $\mathrm{Gm}$ a través del núcleo IMS para cualquiera de los productos IPTV requeridos por el UE. El contenido multimedia por ejemplo puede ser transmitido vía unicast o multicast sobre la interfaz Xd desde la función de entrega de medios
(MDF); las solicitudes SIP también pueden ser transmitidas del SCF al MCF mediante el núcleo IMS usando la interfaz y2. La combinación específica de los protocolos SIP y RTSP permiten proveer adicionalmente el servicio de CoD (call of duty) donde el mensaje SIP INVITE se utiliza para la configuración de la sesión pero el control de flujo de medios se maneja a través de la interfaz Xc usando RTSP [7] [9].

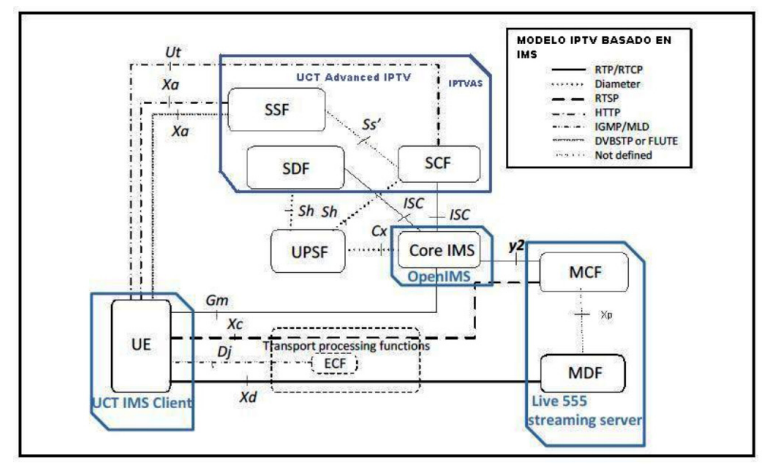

Figura 1. Arquitectura IPTV/IMS

\subsubsection{El equipo de usuario}

El UE o equipo del usuario puede ser un STB (set top box) o un computador con un cliente IMS instalado, y es el encargado de iniciar los mensajes de control y señalización dentro del sistema IPTV. El UE permite al cliente el acceso y visualización de los servicios y contenido ofrecidos por el sistema IPTV.

En el bloque funcional de transporte se encuentran dos subsistemas definidos por la arquitectura NGN, que son el de admisión y el control de recursos (RACS) que tiene la función de reservar y asignar los elementos necesarios para la trasmisión multimedia; el sistema de acoplamiento a la red (NASS) es responsable de asignar direcciones IP, P-CSCF, y demás configuraciones a los UE. El núcleo IMS se encuentra entre la capa de servicio y transporte de la arquitectura y es el que permite la convergencia de redes móviles y fijas, permite una administración centralizada de usuarios y recursos, además administra y transporta todos los mensajes de organización, iniciación de servicio y autenticación [7] [9]. 


\subsubsection{Servidor de aplicación SIP de IPTV (IPTVAS)}

Todas las aplicaciones IMS provienen de un servidor SIP, que puede simplificar enormemente la construcción de prestaciones a través del uso del service creation environments (SCEs) que permite a los desarrolladores concentrarse en el sistema de negocio abstrayéndose de toda la infraestructura de red y por ende simplificando la generación de aplicaciones [6] [7] [8]. La función de descubrimiento de servicios (SDF) genera la información de los productos disponibles para el usuario, permitiendo así entregar un contenido IPTV personalizado. La función de selección (SSF) contiene información de servicios disponibles a los que el UE tiene acceso, como por ejemplo guías de programas de televisión, vídeo a la carta y en vivo. La función de control (SCF) es un SIP AS que proporciona los parámetros de la red que el UE requiere para activar el servicio IPTV incluyendo el canal de entrega y control de contenido, también es responsable de la autorización de productos durante el inicio y modificación de la sesión. Esta autorización incluye la comprobación de los perfiles de usuario para determinar el nivel de privilegios que posee [10].

\subsubsection{Servidor de distribución de multimedia}

Está compuesto por la función de control (MCF) y entrega (MDF) del contenido multimedia, mediante una relación maestro esclavo. El MCF se encarga de la supervisión del estado de la MDF y es responsable de controlar la señalización con el SCF mediante el núcleo IMS. La función principal del MDF es la entrega de los medios multimedia a la UE a través de los métodos de transmisión uniscast y multicast. También realiza la codificación y/o la transcodificación de los sistemas de comunicación para los diferentes formatos requeridos [10], [4].

\subsubsection{Núcleo IMS}

Constituyen los elementos básicos y claves de todas las arquitecturas IMS/NGN. El núcleo IMS es el modulo de control de la arquitectura IMS, y es quien administra los roles y el almacenamiento de datos de los usuarios para brindar las funciones de control de decisiones, autenticación en la red, gestión de roaming, movilidad y control de la calidad del servicio. Está constituido por un conjunto de funciones de control de llamada de sesión IMS (CSCF) y un servidor de abonado local HSS que generan, procesan y transportan mensajes de señalización SIP, mensajes de autenticación y registros del protocolo DIAMETER o XCAP. El conjunto de funciones de control de la sesión de llamada (CSCF), está encargado de establecer la sesión multimedia con y entre los suscriptores, y se compone de cuatro funciones principales que son P-CSCF, S-CSCF, I-CSCF y HSS y que se explican a continuación.

- Proxy - CSCF (P-CSCF): es el primer punto de contacto de los usuarios con el núcleo IMS, su función principal es transportar los mensajes de señalización entre los clientes y el resto del núcleo IMS y el sistema IPTV.

- Serving - CSCF (S-CSCF): es el modo principal dentro del proceso de señalización, utiliza el protocolo DIAMETER para descargar el perfil del usuario, permitiendo conocer la dirección IP del terminal del usuario y la dirección SIP.

- Interrogantinng - CSCF (I-CSCF): realiza consultas al HSS para obtener la dirección del S-CSCF y asignarlo al cliente que realiza el registro. También es utilizado para ocultar la red interna del mundo exterior.

- Servidor de suscripción local (HSS): Es equivalente a la función de servicio de perfil (UPSF), es una base de datos central, que contiene todos los datos de los clientes e información de subscripción relacionada con los perfiles que poseen [6] [7] [8].

\subsection{Protocolos y tecnologías IPTV sobre IMS en IPv6}

La pila de los principales protocolos utilizados en la arquitectura de IPTV sobre IMS es mostrada en la figura 2 soportada en el modelo OSI. 


\begin{tabular}{|c|c|}
\hline APLICACIÓN & RTSP/DNSV6/DHCPV6 \\
\hline PRESENTACIÓN & H.264 \\
\hline CAPA DE SESIÓN & SIP/SDP/RTCP/RTP \\
\hline CAPA DE TRANSPORTE & TCP/UDP \\
\hline CAPA DE RED & IPV6/PIM-SM/MLD/DIAMETER \\
\hline CAPA DE ENLACE & MPLS \\
\hline CAPA FISICA & ETHERNET \\
\hline STB \\
\hline
\end{tabular}

Figura 2. Pila de Protocolos de IPTV

\section{METODOLOGÍA PARA LA IMPLEMENTA- CIÓN DE IPTV SOBRE IMS EN REDES IPV6}

De acuerdo a la exploración temática realizada sobre cada una de las arquitecturas y protocolos de comunicaciones involucrados en la plataforma IPTV/IMS/IPv6, en la figura 3 se segmento el desarrollo de la metodología e implementación de esta estructura en cuatro bloques o áreas funcionales que incluyen:

- Un bloque de IPTV

- Un bloque del núcleo IMS

- Un bloque de usuario final

- Un bloque de Red

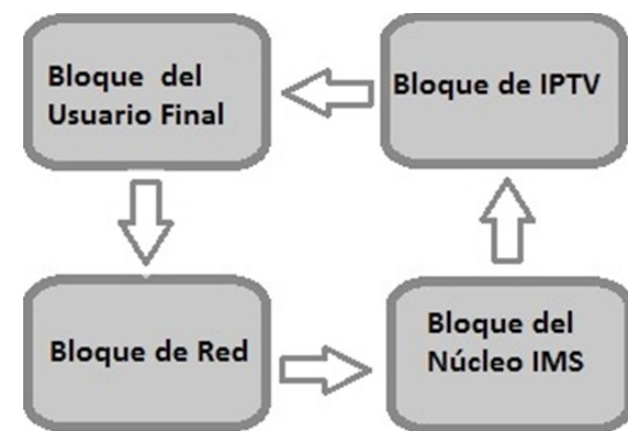

Figura 3. Bloques Funcionales de IPTV sobre IMS

\subsection{Identificación y selección de las funciones básicas que presta la plataforma de IPTV sobre IMS en redes IPV6}

Debido a las limitaciones de hardware y software se hace necesario especificar los servicios básicos prestados por el núcleo contenido de IPTV, IMS y la red de acceso. A continuación se describen los componentes básicos que prestaría cada bloque funcional en caso de implementarse.

\subsubsection{Servicios básicos del bloque de IPTV}

Es el que soporta el servicio de video sobre demanda, y los componentes que lo soportan son el IPTV AS (IPTV application server y el media server).

\subsubsection{Servicios básicos del bloque del núcleo IMS}

Incluye el núcleo IMS y es el que administra el núcleo IMS desde una interfaz web. Los elementos hardware y software que lo soportan son el P-CSCF (Proxy - Call Session Control Function), I-CSCF (Interrogating - Call Session Control Function), S-CSCF (Serving - Call Session Control Function), y el HSS (Home Suscriber Server).

\subsubsection{Servicios básicos del bloque del usuario final}

Determina la capacidad de atender las solicitudes de contenido bajo demanda directamente desde el cliente IMS, y su componente principal es el usuario final.

\subsubsection{Servicios básicos del bloque de red}

Incluye elementos de consulta DHCP (protocolo de control de host dinámico) a nivel de IPv6 (a través de un conmutador o encaminador) y da soporte a las consultas DNS (Servicio de Nombres de Dominio) generadas mediante un servidor.

\subsection{Propuesta de una la metodología para la implementación de la plataforma IPVT sobre IMS en redes IPv6}

El diseño de la metodología se construyó a partir de la exploración del estado del arte de sistemas IPTV sobre IMS para redes IPv6 (aunque la información es muy escasa) incluyendo el hardware y el software más adecuado para su posible implementación, teniendo en cuenta que las aplicaciones lógicas fueran de libre distribución. En la figura 4 aparece a manera de diagrama de flujo la metodología para una estructura de red LAN, seguida de la explicación 
de los principales bloques que lo componen.

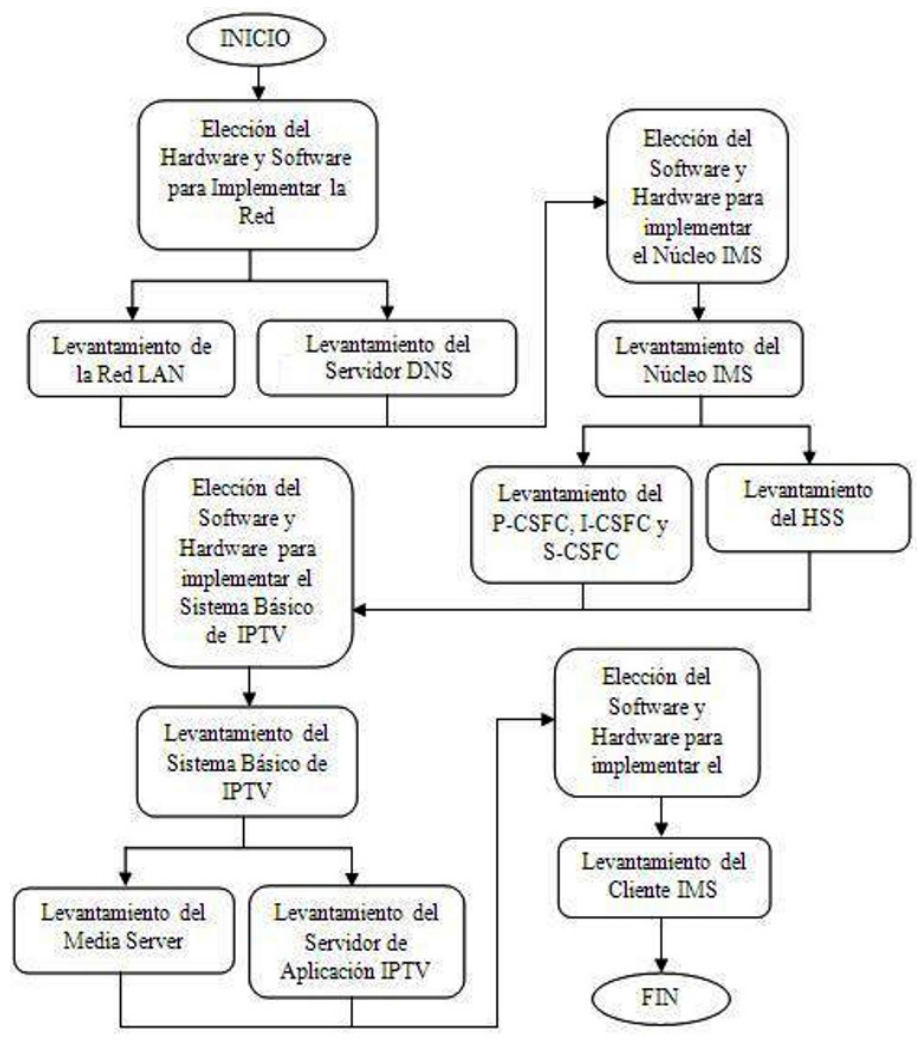

Figura 4. Metodología para la implementación de la plataforma de IPTV sobre IMS

\subsubsection{Elección del software y hardware para la implementación de la red}

En la tabla 1 se genera el posible direccionamiento IPv6 a ser configurado asociado con el correspondiente nombre de dominio para cada uno de los componentes y/o servidores.

Para la construcción de la red de área local solo se requiere de la utilización de un DCE (que es un equipo de conmutación de datos) encargada de la interconexión de los servidores y los clientes.

Tabla 1. Direccionamiento IPv6 y asociación DNS

\begin{tabular}{|c|c|c|}
\hline COMPONENTE & DIRECCIÓN IP & NOMBRE DE DOMINIO \\
\hline Servidor DNS & fec0:0:1:1:0206:5bff:f354:feb2/64 & dns.udistrital.edu.co \\
\hline Cliente IMS & fec0:0:1:1:0218:deff:fe05:558e/64 & ue.udistrital.edu.co \\
\hline P-CSCF & fec0:0:1:1:0216:36ff:fe83:96af/64 & pcscf.udistrital.edu.co \\
\hline I-CSCF & fec0:0:1:1:0216:36ff:fe83:96af/64 & icscf.udistrital.edu.co \\
\hline S-CSCF & fec0:0:1:1:0216:36ff:fe83:96af/64 & scscf.udistrital.edu.co \\
\hline HSS & fec0:0:1:1:0216:36ff:fe83:96af/64 & hss.udistrital.edu.co \\
\hline IPTV AS & fec0:0:1:1:021f:d0ff:fe61:b083/64 & ipvt.udistrital.edu.co \\
\hline Media Server & fec0:0:1:1:021f:d0ff:fe61:b083/64 & media.udistrital.edu.co \\
\hline
\end{tabular}




\subsubsection{Levantamiento del servidor DNS}

La implementación del servidor DNS debiera estar soportada en el software BIND (Berkeley Internet Name Domain) sobre un sistema de libre distribución. La selección de BIND radica en que es uno de los más consolidados y de más amplia utilización en desarrollos de este tipo, además de ser código abierto y con el soporte necesario [15] [8].

\subsubsection{Elección del software y hardware para la implementación del núcleo IMS}

Todo el desarrollo del núcleo IMS debe ser realizada sobre el sistema operativo GNU/Linux donde las características mínimas del hardware deben estar soportadas sobre un procesador Intel Core 4 Duo de $2.00 \mathrm{GHZ}$ y memoria RAM de 8 GB. En cuanto el software para dar soporte a la última versión del protocolo IP se recomienda el uso de Open IMS Core, que da las herramientas necesarias para implementar los cuatro componentes del núcleo IMS y que por recomendación de [3] debieran ir asociados dentro de un mismo servidor y no de forma separada.

\subsubsection{Levantamiento del núcleo IMS}

Las entidades que componentes este core como se ha mencionado son el P-CSCF, I-CSCF, SCSCF y HSS, que han sido implementadas por el proyecto de código abierto Open IMS Core a cargo del FOKUS (Fraunhofer Institute for Open Communication Systems) (FOKUS) con fines de investigación. Se recomienda la utilización de este proyecto ya que es la única implementación de un núcleo IMS de código abierto que existe en el momento. Ella presenta todas las características y funciones requeridas para poder soportar todos los componentes que conformarán un futuro servicio de IPTV, donde su instalación y configuración es encontrada en [16].

\subsubsection{Elección del software y hardware para la implementación del sistema básico de IPTV}

El desarrollo del sistema puede estar incluido sobre una plataforma como Debian 6 soportado en un servidor con un procesador Intel Core 4 Duo de 2.00 GHZ y memoria RAM superior a los 8 GB. En cuanto el software sería adecuado el uso del Live 555 Media Server, ya que da la opción de incluir el Media Server; y para implementar el IPTV AS se podría usar la aplicación UCT Advanced IPTV [13] [10].

\subsubsection{Levantamiento del sistema IPTV básico}

Como se mencionó en el anterior ítem, este bloque está formado por el IPTVAS y el Media Server. El primero de ellos corresponde al servidor de aplicación IPTV (IPTVAS), que debiera ser implementado a partir del proyecto desarrollado por el Communications Research Group de la Universidad de Cape Town llamado UCT Advanced IPTV, ya que esta aplicación permite la adición de un servicio de aplicaciones basado en SIP, diseñado para IPTV [13] [10]. Para la instalación y configuración de IPTVAS se podría recurrir a las especificaciones mostradas en [17]. No obstante es importante destacar que este aplicativo es nativo solo para IPv4; de requerirse la inclusión de IPv6 es necesario la instalación de un complemento que fue desarrollado por el Instituto Nacional de Polonia y la universidad Tecnológica de Gdansk que puede ser descargado de [18].

El segundo componente que se agregará en esta etapa es el Media Server y para ello se debe instalar el VLC SERVER (VideoLan) documentado en [13] [10]. El VLC es capaz de transmitir streaming de video a través del protocolo RTSP. Para la instalación y configuración del Media Server es recomendable seguir la guía que ofrece el 555 Media Server en [19].

\subsubsection{Elección del software y hardware para la implementación del ciente IMS}

El sistema operativo a ser usado puede ser cualquiera que reuna las condiciones del GNU/ Linux y el hardware como mínimo se recomienda que esté formado por un es un portail con un procesador Intel Dual Core de $1.86 \mathrm{GHZ}$ y memoria RAM de 2 GB con el fin de brindar movilidad. En cuanto el software se podría usar el 
UCT IMS Client [2] [13] que es compatible con los demás elementos que componen el sistema.

\subsubsection{Levantamiento del cliente IMS}

En cuanto al cliente, es necesario configurar una cuenta SIP personalizada para acceder al Sistema IMS. Esto se hace utilizando la interfaz Web de configuración del HSS que debe ser levantada para estos fines y para ello, se debe crear una suscripción IMS, luego una identidad privada de usuario y una identidad pública. Igualmente es requerido levantar un cliente IMS con el UCT IMS Client que permita registrarse en el sistema para solicitar canales del futuro servicio IPTV. La ventaja de este software radica en su compatibilidad con el proyecto Open IMS Core del Instituto FOKUS y de poseer características útiles para la implementación de un laboratorio de IPTV, entre las que se incluyen la capacidad de solicitar canales de IPTV, incluir botones PLAY, PAUSE y STOP para controlar sesiones multimedia RTSP. Para su instalación y configuración es necesario basarse en la guía ofrecida en [20].

\subsection{Topología física de IPTV sobre IMS en redes IPv6}

Finalmente en la figura 5 se presenta la posible plataforma que se usará para probar la metodología propuesta a lo largo de este artículo y que será desarrollada en la siguiente etapa del proyecto de aplicación y que tiene como finalidad ajustar el modelo metodológico inicial.

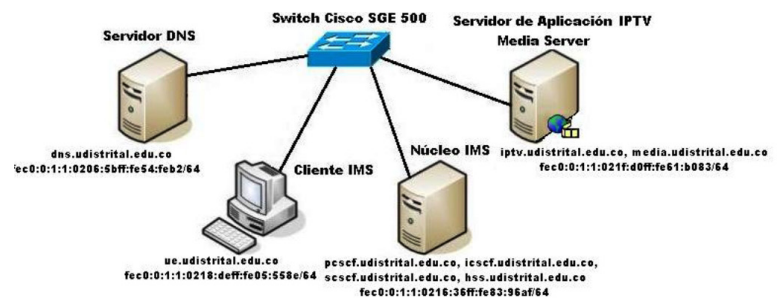

Figura 5. Plataforma de IPTV sobre IMS en redes IPv6.

\section{CONCLUSIONES}

Este artículo permite plasmar y estudiar las tendencias de los nuevos estándares que han dado lugar a la evolución e implementación de IPTV sobre IMS en redes IPv6.

La implementación de IPTV sobre IMS con software libre permite el fácil acceso a toda la información y documentación para la instalación y configuración de cada uno de los servidores, aunque en algunos casos su soporte es escaso.

Dentro de este trabajo se describieron las arquitecturas y componentes necesarios que soportan IPTV con el fin de desarrollar una primera aproximación a generar una metodología que sirva como base para el diseño e implementación de una plataforma IPTV sobre IMS para IPv6, en donde dentro del desarrollo de la metodología y exploración temática se logró encontrar entre otros detalles que el envío de las credenciales se realiza mediante el protocolo SIP de forma insegura ya que la señalización viaja sin cifrar, permitiendo realizar un ataque del tipo Man in The Middle y obtener la información enviada y/o solicitada por el cliente IMS. Para evitar este tipo de ataques se debiera adicionar al proceso de señalización un estándar de transporte seguro como el que incluye TLS para tener un mecanismo de seguridad y autenticidad en el establecimiento de la sesión.

De otro lado el acceso al contenido de IPTV se realiza a través del protocolo RTP el cual no posee ningún mecanismo de cifrado y autenticación, por lo anterior es necesario usar el protocolo SRTP definido en el RFC 3711 con el fin de establecer niveles de seguridad en la transmisión de la multimedia, que permitan proporcionar cifrado, autenticación del mensaje, integridad, y protección. 
[1] O. Friedrich, R. Seeliger, S. Arbanowski; Interactive and personalized Services for an open IMS-based IPTV infrastructure, Networking, ICN-2008, Seventh International Conference on, pp 302-307, Mexico, 2008.

[2] S. Janikowski, J. Mongay; On extending open source IMS platform for integrated IPTV and VoIP services over IPv6, Telecommunications Network Strategy and Planning Symposium (NETWORKS), 2012 XVth International, pp 1-6, Rome, 2012.

[3] I. Mkwawa, E. Jammeh, L. Sun, A. Khan, E. Ifeachor; Open IMS Core with VoIP quality adaptation, Fifth International Conference on Autonomic and Autonomous Systems, pp 295-300, Spain, 2009.

[4] E. Mikoczy, D. Sivchenko, V. Rakocevic; IMS based IPTV services - Architecture and Implementation, Proceedings of the 3rd international conference on Mobile multimedia communications (MobiMedia 2007), pp 1-17, London, 2007.

[5] A. Punchihewa, A. Silva; Tutorial on IPTV and its Latest Developments, Information and Automation for Sustainability (ICIAFs), 2010 5th International Conference on, pp 45-50, Colombo, 2010.

[6] M. Qadeer, H. Afaq, A. Juned, A, S. Waheed; IMS network architecture, Conference on future computer and communication IMS network architecture, pp 50-55, 2010.

[7] J. Rittwik, J. Murray, C. Rice; IMS-TV: An IMS-Based Architecture for Interactive, Personalized IPTV, Communications Magazine, IEEE, vol 45, issue 11, pp 156163, 2008.

[8] K. Rottmann, K. Rainer; Diseño e implementación de un laboratorio de IPTV, medición y gestión, Tesis de pregrado, Universidad de Chile, Chile, 2010.

[9] E. Mikoczy, S. Telekom, D. Sivchenko, B. Xu, D. Telekom, J. Moreno; IPTV
Services over IMS: Architecture and Standardization. [En línea], consultado en Septiembre 17 de 2012, disponible en: http://www.tele.pw.edu.pl/ mareks/eims/ IPTV-Services-over-IMS.pdf.

[10] R. Spiers, R. Marston, R. Good, N. Ventura; The UCT IMS IPTV initiative, Next Generation Mobile Applications, Services and Technologies, NGMAST '09 Third International Conference on, pp 503-508, Cardiff (Wales), 2009.

[11] J. Torres, R. Ramírez, D. López; Estado del arte de IPTV y consideraciones técnicas para su migración a IPv6, Revista Redes de Ingeniería, vol. 2, no. 1, pp. 4564, 2011.

[12] Over 2.1 million live IPTV subscribers being served by Alcatel-Lucent platform (iLocus). [En línea], consultado en Enero 20 de 2012, disponible en: http://www. ilocus.com/2008/05/over-21-million-liveiptv-subs.html.

[13] B. Villanueva, D. López; A Proposal for implementing an IMS-based IPTV system over an IPv6/MPLS network using open source software, The Seventh International Conference on Digital Society (ICDS 2013), pp 48-52, France, 2013.

[14] M.Zahid, M.Qadeer,A.Iqbal; Deployment of IPTV over IMS architecture, Internet Multimedia Services Architecture and Applications (IMSAA), 2nd International Conference on, pp 1-6, Bangalore, 2008.

[15] Configuración del servidor DNS. [En línea], consultado en Abril 28 de 2013, disponible en: http://man-es.debianchile. org/bind.html.

[16] Open source IMS. [En línea], consultado Marzo 11 de 2013, disponible en: http:// www.openimscore.org/node/143.

[17] IMS Client, how to setup UCT advanced IPTV. [En línea], consultado en Enero 7 de 2013, disponible en: http://uctimsclient. berlios.de/uctviptv_advanced_howto. html.

[18] IMS_IPTVv6_VoIPv6. [En línea], 
consultado Febrero 19 de 2013, disponible en: ftp://ftp.itl.waw.pl/IMS_IPTVv6_ VoIPv6.

[19] The LIVE555TM media Server. [En línea], consultado en Diciembre 9 de 2012, disponible en: http://www.live555. com/mediaServer/.
[20] UCT IMS client, download and install the UCT IMS client package. [En línea], consultado en Noviembre 28 de 2013, disponible en: http://uctimsclient.berlios. de/uctimsclient_on_ubuntu_howto.html 